\title{
50 AÑOS DE LA CONVENCIÓN DE NUEVA YORK: IMPLICANCIAS PARA AMÉRICA LATINA
}

\author{
Jonathan C. Hamilton*
}

El año 2008 marcó el $50^{\circ}$ aniversario de la Convención sobre el Reconocimiento y Ejecución de las Sentencias Arbitrales Extranjeras de 1958 (en adelante la "Convención de Nueva York" ${ }^{\prime}{ }^{1}$ El presente artículo explica cómo el marco jurídico del arbitraje comercial en América Latina ha evolucionado durante este período, y compara la Convención de Nueva York con la Convención Interamericana sobre Arbitraje Comercial Internácional de 1975 (la "Convención de Panamá"). ${ }^{2}$ Este artículo también discute la aplicación de las Convenciones de Nueva York y Panamá en jurisdicciones de la región y las implicancias para América Latina de una propuesta para modificar la Convención de Nueva York.

\section{Tratados De Arbitraje Internacional}

En el contexto del arbitraje comercial internacional en América Latina existen dos principales tratados que actualmente han sido ratificados por casi todos los países de América Latina: la Convención de Nueva York y la Convención de

* Socio, White \& Case LLP, Washington, D.C.; Presidente de la Iniciativa para las Américas del Institute for Transnational Arbitration (ITA). Este articulo está basado en parte en una presentación realizada por el autor en el Seminario Internacional de la Nueva Ley de Arbitraje realizado en Lima, Perú, en agosto de 2008 .

1. La Convención sobre el Reconocimiento y la Ejecución de las Sentencias Arbitrales Extranjeras, 10 de junio de 1958, 21 U.S.T. 2517,330 U.N.T.S. 38.

2. Organización de Estados Americanos [OEA], Convención Interamericana sobrc Arbitraje Comercial Internacional, 30 de enero de 1975,1438 U.N.T.S. 245. 
Panamá. ${ }^{3}$ El Apéndice A contiene una sinopsis de la ratificación de estas convenciones por países de América Latina. ${ }^{4}$

La Convención de Nueva York no fue inmediatamente aceptada por los países de América Latina después de su origen en el año 1958. Tal como lo demuestra el Apéndice B, para su $25^{\circ}$ aniversario en 1983, la Convención de Nueva York solamente había sido ratificada por seis países de América Latina.

En el mismo período, en un esfuerzo por promover el arbitraje comercial en la región, la Organización de Estados Americanos ("OEA") llevó a cabo su Primera Conferencia Interamericana Especializada sobre Derecho Internacional Privado en la República de Panamá en 1975. Al final de la Conferencia, "deseosos de concluir una convención sobre arbitraje comercial internacional", los países de la OEA promulgaron la Convención de Panamá.

La Convención de Panamá significó un paso adelante hacia la aceptación del arbitraje comercial internacional en América Latina ${ }^{5}$ Negociada durante varios años, ésta fue elaborada con

3. Adicionalmente a las Convenciones de Nueva York y Panamá, algunos paises de América Latina también han ratificado otras convenciones regionales. Algunos ejemplos son la Convención Interamericana sobre Eficacia Extraterritorial de las Sentencias y Laudos Artitrales Extranjeros (la "Convención de Montevideo") y el Protocolo de Las Leñas de 1992 adoptado por los países miembros del Mercado Común del Sur ("MERCOSUR").

4. El Apéndice A es un extracto del Compendio Sobre Arbitraje en América Latina. Ver Jonathan C. Hamilion, The Compendium of Latin American Arbitration Law, INT'L DiSP. Q. (Verano 2009). El Compendio está basado en un análisis de diversas fuentes y solamente puede ser reproducido o traducido con atribución al autor. Este extracto se enfoca en arbitraje comercial en América Latina. Para un resumen sobre arbitraje de inversiones en América Latina, ver Jonathan C. Hamilton, $A$ Decade of Latin American Investment Arbitration, LATIN AMERICAN INVESTMENT TREATY ARBITRATION: THE CONTROVERsies and Conflicts 69 (Mary H. Mourra \& Thomas E. Carbonneau eds., 2008) (referente al arbitraje de inversiones en América Latina).

5. Una característica distintiva del arbitraje ha sido el marco de ejecución de tratados - algo que falta con respecto a la ejecución de sentencias de cortes extranjeras. Sin embargo, recientemente un pais de América Latina (México) fue el primero en racificar la Convención de la Haya sobre Acuerdos de Elección de Foro ("Convención sobre Elección de Foro"). México ratificó la Convención elu septiembre de 2007 , la cual fue firmada por los Estados Unidos en enero de 2009 y por la Unión Europea en abril de 2009. A pesar de que previamente no habia un marco general sobre tratados internacionales para el reconocimiento de sentencias de cortes, la Convención sobre Elección de Foro busca brindar certeza con respecto a la ejecución de sentencias de litigios. Intenta lograr esta meta exjgiendo a los estados contratantes reconocer y ejecutar sentencias adoptadas por las cortes elegidas, salvo ciertas excepciones y en la medida que no otorguen a la parte afectada una compensación mayor que la de los daños efectivos. La adopción de la Convención de Elección de Foro por otros paises de América Latina puede afectar la solución de controversias en la región. 
posterioridad de la Convención de Nueva York. Un objetivo primordial de la Convención de Panamá fue el de corregir las deficiencias percibidas en las leyes de arbitraje domésticas de países de América Latina, estableciendo los requisitos para acuerdos arbitrales válidos, el procedimiento para el reconocimiento y ejecución de laudos arbitrales, y otros asuntos relacionados con el procedimiento arbitral. Como demuestran los Apéndices A y C, 6 la Convención de Panamá ha sido actualmente ratificada por un total de diecisiete países, incluyendo dieciséis países de América Latina y los Estados Unidos ${ }^{7}$. Sin embargo, no todos los países de América Latina han ratificado este tratado.

Mientras tanto, tal como se demuestra en los Apéndices A y $C$, la Convención de Nueva York ha sido actualmente ratificada por un total de 144 países, incluyendo diecinueve países de América Latina. En general, la ratificación de la Convención de Nueva York por los países de América Latina ha seguido el ritmo de ratificación de la Convención de Panamá - por ejemplo, en 1995, quince países de América Latina habían ratificado la Convención de Panamá, mientras que la Convención de Nueva York había sido adoptada por trece estados en la región. ${ }^{8}$

6. El Apéndice A es un extracto del Compendio Sobre Arbitraje en América Latina. Ver Jonathan C. Hamilton, The Compendium of Latin American Arbitration Law, InT'L DISP. Q. (Verano 2009). El Compendio está basado en un análisis de diversas fuentes y solamente puede ser reproducido o traducido con atribución al autoc. Este ensayo se enfoca en arbitraje comercial en América Latina. Para un resumen sobre arbitraje de inversiones en América Latina, ver Jonathan C. Hamilton, A Decade of Latin American Investment Arbitration, LATIN AMERICAN INVESTMENT TREATY ARBITRATION: THE CONTROVERSIES AND CONFLICTS 69 (Mary H. Mourra \& Thomas E. Carbonneau eds., 2008) (refetente al arbitraje de inversiones en América Latina).

7. Ver Sistema de Información sobre Comercio Exterior de la OEA, Solución de Controversias: Arbitraje Comercial, La Convención Interamericasa sobre Arbitraje Comercial Internacional, disponible en http:/www.sice.oas.org/dispute/comart/iacac /acac2e.asp (listando los países signatarios). En relación con la historia de la negociación de la Convención de Panamá, ver OEA, Proyecto Convención Interamericana Sobre Arbitraje Comercial Internacional, Actas y Documentos de la Conferencia Especializada Interamericana Sobre Derecho Internacional Privado, vol. 2, OEA Doc. Ser.K/XXI. /CIDLP-64, 15 de enero de 1975.

8. Además de ratificar las Convenciones de Nueva York y Panamá, muchos paises de América Latina también han modificado sus legistaciones de arbitraje domésticas para promover el tipo de arbitraje comercial contemplado en estos tratados. Los cambios en leyes arbitrales en América Latina fueron parte de reformas legates realizadas principalmente en los años noventa, y esos cambios se basaron en todo o en parte en la Ley Modelo de la Comisión de las Naciones Unidas para el Derecho Mercantil Internacional ("Ley Modelo de la CNUDMI"). Ver, por ejemplo, Jonathan C. Hamilton, International Arbitration as a Component of Latin American Reforms. The Case of Peru (1997). 


\section{Comparación de las Convenciones de NUEVA YORK Y PANAMÁ}

Las Convenciones de Nueva York y Panamá promueven el respeto por la ejecución de acuerdos arbitrales válidos y el reconocimiento y ejecución de laudos arbitrales que cumplen con ciertos requisitos mínimos. Al mismo tiempo, ambos tratados permiten que las cortes se nieguen a reconocer y ejecutar laudos impropios. Por ejemplo, ambos tratados permiten a las cortes rechazar el reconocimiento y la ejecución cuando, entre otras cosas, el procedimiento arbitral fue contrario al acuerdo de las partes, o cuando el laudo ha violado los derechos al debido proceso de la parte en contra de la cual se invoca la sentencia arbitral.

La Convención de Panamá comparte muchas disposiciones importantes con la Convención de Nueva York, además del principal objetivo de proteger y promover el arbitraje internacional. No obstante, existen diferencias claves entre las dos convenciones. Algunas de estas diferencias están relacionadas con el ámbito de aplicación y las reglas de procedimiento de las dos convenciones. Otras diferencias tienen que ver con la obligación de las cortes de obligar a las partes a ir a arbitraje, así como los requisitos que las partes deben cumplir para obtener el reconocimiento y la ejecución de laudos arbitrales.

Otra diferencia entre las dos convenciones es la regla especial supletoria establecida en el Artículo 3 de la Convención de Panamá, la cual establece que "a falta de acuerdo expreso entre las partes el arbitraje se llevara a cabo conforme a las reglas de procedimiento de la Comisión Interamericana de Arbitraje Comercial. "9 Esta medida tuvo como objetivo promover un marco legal regional para el arbitraje. En este sentido, el Artículo 3 contempla crear un sistema de arbitraje administrado por una institución enfocada en arbitraje para las Américas, y consecuentemente reducir

9. Ver Convención de Panamá, ut stupra nota al pie 1, Art. 3. 
la necesidad de intervención judicial y minimizar incertidumbre en los procedimientos arbitrales. Cabe destacar que desde el 2002, el Centro Internacional para la Resolución de Disputas ("ICDR", por sus siglas en inglés) del American Arbitration Association ("AAA", por sus siglas en inglés) ha tenido un acuerdo con la Comisión Interamericana de Arbitraje Comercial ("CIAC") para administrar arbitrajes presentados ante la CIAC. ${ }^{10}$

Por otro lado, un número de temas tratados en la Convención de Nueva York no se encuentran presentes en la Convención de Panamá. A diferencia del Artículo 1 de la Convención de Nueva York, la Convención de Panamá no especifica el ámbito de su aplicación -tanto con respecto al asunto materia de arbitraje o la nacionalidad de las partes-. La Convención de Panamá tampoco incluye la disposición de reciprocidad establecida en el Artículo I(3) de la Convención de Nueva York, que le permite a los estados requerir que laudos arbitrales sean adoptados en el territorio de otro estado contratante. ${ }^{11}$ También la Convención de Panamá deja abierto para una amplia interpretación la cuestión de cuando un arbitraje es considerado "internacional." La Convención de Panamá tampoco requiere que las cortes de los estados miembros lleven a las partes a arbitraje después de determinar que las partes han firmado un acuerdo arbitral válido. Finalmente, la Convención de Panamá no contempla los requisitos formales que exige la Convención de Nueva York en su Artículo IV, tales como la presentación del original o una copia debidamente certificada del laudo original y del acuerdo arbitral original, o una traducción certificada en caso fuese necesario.

10. Ver Luis M. Martinez, Are We There Yet?, en Arbitration Review of THE AMERICAs (2009).

11. Ver Convención de Nueva Yotk, ut supra nota al pie 1, Art. I(3). 


\section{Las Convenciones Arbitrales y la Práctica arbitral}

Un asunto fundamental, en teoría, es cual de las dos convenciones es aplicable en un procedimiento de ejecución. Con respecto a la interrelación entre las Convenciones de Nueva York y Panamá, el Artículo VII de la Convención de Nueva York dispone que:

Las disposiciones de la presente Convención no afectarán la validez de los acuerdos multilaterales o bilaterales relativos al reconocimiento y la ejecución de las sentencias arbitrales concertados por los Estados Contratantes ni privarán a ninguna de las partes interesadas de cualquier derecho que pudiera tener a hacer valer una sentencia arbitral en la forma y medida admitidas por la legislación o los tratados del país donde dicha sentencia se invoque. ${ }^{12}$

La Convención de Panamá no contiene una disposición de compatibilidad como ésta. Para algunos comentaristas, esta discrepancia sugiere que la Convención de Panamá debe aplicarse en casos de conflicto entre las dos convenciones, especialmente dado que la Convención de Panamá es un instrumento enfocado en la región, y es posterior a la Convención de Nueva York. ${ }^{13}$ En otros contextos, se da preferencia al tratado más favorable al reconocimiento y ejecución del laudo arbitral. ${ }^{14}$

En teoría, en la mayoría de los casos las Convenciones de Nueva York y Panamá pueden dar lugar a resultados similares. Los casos que se describen a continuación demuestran cómo coexisten en la práctica estas dos convenciones. A continuación realizamos un análisis comparativo de una selección de leyes y precedentes de distintos países en América Latina.

12. Convención de Nueva York, $u$ supra nota al pie 1, Art. Vil.

13. Ver, por ejemplo, Jan Kleinheisterkamp, InTERNATIONaL COMmerclal ARBITRATION IN LATIN AMERICA 27 (2005) ("Hay buenas razones en favor de la solución simple de la regla general de conflicto de tratados lex specialis derogate generali.") (traducido al español). En reconocimiento al hecho de que la Convención de Nueva York permite otros acuerdos y considera problemas locales, Kleinheisterkamp razona "[s]i el arbitraje es genujnamente interamericano, la Convención de Panamá prevalecerá como lex specialis sobre la Convención [de Nueva York]." Idem.

14. Ver, por ejemplo, Sección 5.2. (explicando la ley peruana de arbitraje del 2008 que sigue esta postura). 


\subsection{Estados Unidos}

En los Estados Unidos, la respuesta sobre qué convención es aplicable para el reconocimiento y ejecución de laudos arbitrales está establecida en la Sección 305 de la Ley de Arbitraje Federal -Federal Arbitration Act (la "FAA"). 15 De acuerdo con la Sección 305:

Cuando se cumplan los requisitos para la aplicación de tanto la [Convención de Panamá] y la [Convención de Nueva Yorkl, la determinación sobre cual Convención es aplicable, a menos que haya acuerdo en contrario, deberá ser establecida de la siguiente manera: (1) Si la mayoría de las partes del acuerdo arbitral son ciudadanos de un estado o estados que han ratificado o accedido a la [Convención de Panamá] y son estados miembros de la Organización de Estados Americanos, se aplicará la Convención de Panamá. (2) En todos los demás casos se aplicará [la Convención de Nueva York]. ${ }^{16}$ (Traducido al español).

La Sección 305 representa un intento por el Comité Judiciario de la Cámara de Representantes de los Estados Unidos de proporcionar un procedimiento uniforme para la aplicación de las Convenciones de Nueva York y Panamá por medio del establecimiento de una clara jerarquía. Las cortes estadounidenses han reconocido la obligación de respetar esta jerarquía impuesta por la Sección $305 .{ }^{17}$

15. Ver, por ejemplo, John P. Bowman, The PANAMA CONVENTION AND ITS IMPLEMENTATION UNDER THE Federal ARBitration ACt 81, nota 239 (2002).

16. Federal Arbitration Act, 9. U.S.C. §305 (2000).

17. Ver, por ejemplo, Banco de Seguros del Estado C. Mutual Marine Offices, Inc., 230 F. Supp. 2d 362, 367, n.4 (S.D.N.Y. 2002), aff'd 344 F.3d 255 (2d Cir. 2003) ("Cuando los requisitos tanto de la Convención Interamericana y de la Convención de Nueva York se cumplen, la Convención Interamericana gobierna si 'una mayoría de las partes en el acuerdo arbitral son ciudadanos del estado 0 estados que han ratificado o accedido a la Convención Interamericana y son estados miembros de la Organización de Estados Americanos.") (traducido al español); Progressive Casualty Ins. c. C.A. Reaseguradora Nacional de Venezuela, 802 F. Supp. 1069, 1074 (S.D.N.Y. 1992), anulado por otros motivos, 99] F.2d 42 (2d Cir. 1993)) ("La Convención de Nueva York y la Convención Interamericana ambas representan un pensamiento legal y político actual en favor del arbitraje como un medio de solución alternativa de disputas. En la sección 305 el Congreso razonablemente consideró decidir entre dos convenciones si las dos aplicaban a un mismo contrato.") (traducido al español). 
Esta jerarquía no existe en las leyes de muchos países de América Latina, y la determinación de qué convención es aplicable recae generalmente en las cortes locales. Es más, incluso cuando las partes han especificado qué convención deberá aplicarse, un comentarista sugiere que "no es seguro que los jueces en América Latina vayan a aceptar la elección especifica de las partes por una u otra Convención -una elección que es permitida por la legislación de implementación de los Estados Unidos-." 18

\subsection{Perú}

El Artículo 74(1) de la nueva Ley de Arbitraje del Perú, promulgada el año 2008, dispone que los laudos extranjeros serán reconocidos y ejecutados de acuerdo con: (a) la Convención de Nueva York; o (b) la Convención de Panamá; o (c) cualquier otro tratado sobre reconocimiento y ejecución de laudos arbitrales del cual sea parte el Perú. Con respecto a discrepancias potenciales entre estos instrumentos, el Artículo 74(2) de la nueva ley sigue al Artículo 129 de la ley de 1996, privilegiando la ejecución de laudos extranjeros en el sentido más amplio posible al disponer que "salvo que las partes hayan acordado algo distinto, el tratado aplicable será el más favorable a la parte que solicite el reconocimiento y ejecución de un laudo extranjero."

Bajo los Artículos 76 y 77 de la nueva ley, el reconocimiento y ejecución de laudos extranjeros en Perú está diseñado como un proceso de dos pasos, donde las cortes deben primero reconocer el laudo en todo o en parte, y luego ordenar su ejecución. Adicionalmente, al igual que el Artículo 129 de la ley de 1996, el Artículo 75 de la nueva ley sigue de cerca las causales para negar el reconocimiento de un laudo previstas en el Artículo 36(1) de la Ley Modelo promulgada por la Comisión de las Naciones Unidas para el Derecho Mercantil Internacional ("CNUDMI") y en el Artículo V de las Convenciones de Nueva York y Panamá.

18. Kleinheisterkamp, ut supfa nota al pie 21, en 28 (traducido al español), 
Otra vez reflejando una postura pro-ejecución, el Artículo 75 de la nueva ley se aplica: (1) cuando no hay un tratado internacional aplicable; o (2) cuando hay un tratado internacional aplicable pero la ley en sí misma es mas favorable para la parte que pretende el reconocimiento de un laudo extranjero, teniendo en cuenta los plazos de prescripción previstos en las leyes peruanas. En el mismo sentido -y reconociendo que el Artículo VII(1) de la Convención de Nueva York señala que no afectará la validez de otros acuerdos multilaterales o bilaterales de los que sean parte los estados contratantes- el Artículo 78 de la nueva ley de arbitraje del Perú va un paso más allá que la ley de 1996, en cuanto a que permite en forma específica a las partes aprovecharse de los derechos mas útiles a sus acuerdos arbitrales y laudos.

\subsection{Chile}

A pesar de que Chile fue uno de los primeros países de América Latina en ratificar la Convención de Nueva York en 1975, el desarrollo de su régimen legal arbitral demoró años en comparación con otros países de la región. ${ }^{19}$ En 2004, sin embargo, Chile promulgó una nueva ley de arbitraje (Ley No. 19.971), basada sustancialmente en la Ley Modelo de la CNUDMI. ${ }^{20}$ Las cortes chilenas han sostenido que la nueva ley rige el procedimiento arbitral respecto de los contratos firmados con anterioridad a su promulgación. ${ }^{21}$

19. Ver, por ejemplo, Carlos Eugenio Jorquiera y Karin Helmlinger, Chile, en InTERnatonaL ARBITRATION IN LATIN AmERJCA 89, 184 (Nigel Blackaby, David Lindsey y Alessandro Spinillo eds., 2002) ("[P]ara que Chile se desarrolle como un posible centro de arbitraje internacional es fundamental la actualización de su legislación arbitral del siglo diecinueve. . ...") (traducido al español).

20. Ver, por ejemplo, Kleinheisterkamp, ut supra nota al pie 21, pág. 8; Jonatlan C. Hamilton et al., Chile: Chilean Appellate Court Rules on Applicability of International Arbitration Law, INT'L Dise. Q. 19 (2007).

21. Jonathan C. Hamilton et al., Commercial Arbitration in Latin America, 1 INT'L DISP. Q. 13, 19 (2007) (citando a D'Arcy Masius Benton \& Bowles Inc. Ltda. c. Otero Lathrop Miguel, Recurso de Hecho, Rol No. 865-2000 (Conte de Apelaciones de Santiggo, Primera Sala, May 26, 2006) (sosteniendo que la ley arbitral chilena de 2004 aplica a disputas que surjan de contratos firmados con antcrioridad a su promulgación). 
La ley arbitral chilena no regula expresamente la relación entre las Convenciones de Nueva York y Panamá, ni especifica alguna preferencia entre ellas. En consecuencia, son las cortes chilenas quienes determinan por sí mismas qué instrumento prevalece en caso de conflicto. Sin embargo, las cortes chilenas generalmente han demostrado respeto hacia los procesos arbitrales, confirmando entre otras cosas que el rol de las cortes locales en procedimientos arbitrales internacionales es limitado. 22

En 2008, por ejemplo, la Corte Suprema de Chile rechazó argumentos presentados en oposición al reconocimiento y ejecución de un laudo emitido en Brasil, señalando que los argumentos de la reclamante excedían el marco de su revisión. ${ }^{23}$ Por el contrario, "[l]a Corte Suprema señaló que el único propósito del procedimiento de ejecución era verificar el cumplimiento de ciertos requisitos mínimos relativos al respeto al orden público, la notificación valida a la parte contra la cual se invoca el laudo, el respeto a los límites de jurisdicción y el carácter definitivo de las decisiones a ser ejecutadas. "24

\subsection{México}

La ley mexicana de arbitraje que se encuentra en el Libro V, Título IV del Código de Comercio fue promulgada en 1993 y sigue de cerca a la Ley Modelo de la CNUDMI. El Artículo 1415 de la ley dispone que la ley del arbitraje prevalece "salvo lo dispuesto en los tratados internacionales de que México sea parte o en otras leyes que establezcan un procedimiento distinto o dispongan que determinadas controversias no sean susceptibles de arbitraje." En este sentido, las Convenciones de Nueva York y Panamá constituyen "la ley suprema de toda la unión" bajo el

22. Ver, por ejemplo, D'Arcy, Rol No. 865-2010 (Chile).

23. Ver Cristián Conejero Roos, The New York Convention in Latin America: Lessons from Recent Court Decisions, Global ARBITRATION REv. (2009), disponible en:

http:/www.globalarbitrationreview.com/handbooks/13/sections/50/chapters/499 (citando a Gold

Nutrition Industria e Comercio c. Laboratorio Garden House S.A . (15 de septiembre de 2008)).

24. Idem. (traducido al español). 
Artículo 133 de la Constitución mexicana y, como tales, son de rango superior a la legislación federal en México. ${ }^{25}$

Como en el caso de Chile, la ley de arbitraje de México no regula expresamente la relación entre las dos convenciones, ni tampoco establece una preferencia entre ambas. Al mismo tiempo, dado que la ley de arbitraje de México incorpora las principales disposiciones de ambas convenciones a través de su adherencia a la Ley Modelo de la CNUDMI, las cortes mexicanas raramente aplican las convenciones mismas en forma directa.

En algunas ocasiones, las cortes mexicanas han basado sus decisiones para ejecutar laudos en las convenciones. En el caso de Nordson Corporation c. Industrias Camer S.A. de C.V., por ejemplo, una parte estadounidense solicitó el reconocimiento y ejecución en México de un laudo arbitral otorgado bajo las reglas de la AAA. ${ }^{26}$ Aunque la ejecución fue concedida en primera instancia, la parte mexicana presentó un amparo. Confirmando la decisión de la corte originaria, la Sexta Cámara Civil del Primer Circuito basó su decisión tanto en la Convención de Nueva York como en la de Panamá. En su decisión, la corte sostuvo que "los fundamentos bajo la Convención de Nueva York eran muy restrictivos y que los jueces tenían permitido revisar el fondo de la decisión contenida en el laudo arbitral."27

\subsection{Argentina}

Sin perjuicio de que Argentina es una de las jurisdicciones más activas dentro del arbitraje comercial, su régimen doméstico ha cambiado muy poco desde el siglo diecinueve. ${ }^{28} \mathrm{De}$ hecho,

25. Ver Claus von Wobeser, Mexico, en INTERNATIONAL ARBITRATION IN LATIN AMERICA, ut supra nota al pie 27, págs. 155, 184 (reconociendo que los tratados internacionales ratificados por México, incluyendo las Convenciones de Nueva York y Panamá, "están situados arriba de la legíslación federal del pais en la jerarquía legal del país, y que obligan tanto a las autoridades como a las personas") (traducido al español). "Estos tratados son ejecutorios sin necesidad alguna de legislación reglamentaria." Idem. (traducido al español).

26. Ver Conejero Roos, ut stipra nota al pie 31.

27. Idem.

28. Ver, por ejemplo, Alessandro Spinillo y Emilio Vogelius, Argentina, in INTERNATIONAL ARBITRATION IN LATIN AMERICA, ut supra nota al pie 27, 22, pp. 22-23 (discutiendo "la urgente necesidad de refor- 
varios intentos por adoptar la Ley Modelo de la CNUDMI en Argentina no han tenido éxito. ${ }^{29}$ Es más, dado que las leyes de arbitraje son anteriores a la ratificación de las Convenciones de Nueva York y Panamá, Argentina no tiene una ley que regule específicamente el procedimiento en casos que traten sobre el reconocimiento y ejecución de laudos extranjeros.

En Argentina, los laudos extranjeros son ejecutados bajo tratados internacionales -tales como las Convenciones de Nueva York y Panamá- de ser éstos aplicables. ${ }^{30}$ A pesar de que las cortes argentinas están obligadas a respetar estos tratados, no existe un procedimiento para resolver discrepancias entre las dos convenciones si las partes mismas no han elegido una de las convenciones. En otros casos, laudos extranjeros son ejecutados bajo el Código de Procedimientos Civil y Comercial de la Nación. ${ }^{31}$ En todos los casos, la ejecución debe ser solicitada en las cortes locales que hubieran sido competentes en caso de no haber existido el arbitraje.

\section{Implicancias de una Propuesta de Modificación de la Convención de Nueva York}

La relación entre la Convención de Panamá y la Convención de Nueva York ha sido materia de cierta fricción, como se refleja en los comentarios de académicos como el Profesor Albert Jan van den Berg, autor de una de las obras principales sobre la Convención de Nueva York. ${ }^{32}$ Hace dos décadas, el Profesor van

mar la ley nacional de arbitraje [en Argentina] que todavia data del siglo diecinueve" y proponiendo la adopciôn de la Ley Modelo de la CNUDMl "que ciertamente mejoraria la seguridad juridica y la predictibilidad del procedimiento arbitral, ayudando a crear un nuevo clima en el cual corporaciones multinacionales que lleven negocios en la región puedan estar dispuestas a aceptar cláusulas de arbitraje internacional especificando arbitraje en Argentina") (traducido al español).

29. Ver Kleinheisterkamp, ut supra nota al pie 21, p. 6 (atribuyendo la falla de los cuatro intentos desde 1990 de introducir la Ley Modelo de la CNUDMI a la falta de interés por parte de la Cámara de Diputados).

30. Ver Spinillo y Vogelius, ut supra nota al pie 27, pp. 55-57 (proporcionando un panorama de la ejecución de laudos extranjeros en Argentina).

31. Idem.

32. Albert Jan van den Berg, THE New YoRK CONVENTION OF 1958: Towards a UNIFORM JUdicial INTERPRETATION (1981).

304 Revista Ecuatoriana de Arbitraje 
den Berg señaló en un artículo publicado en 1989 que "[l]a hostilidad tradicional hacia ... el arbitraje internacional en América Latina parece estar disminuyendo," y se preguntó si las convenciones son compatibles y "pueden co-existir." 33 Después de analizar las diferencias entre las dos convenciones, el Profesor van den Berg concluyó que la respuesta a la pregunta de compatibilidad era "un reservado si." 34

Observando como las Convenciones han sido interpretadas y aplicadas por tribunales arbitrales y cortes locales, el Profesor van den Berg comentó sobre la Convención de Nueva York en 1999: "[i]f it ain't broke, don't fix it (si no está rota, no la arreglen)." 35 Luego de una década de nuevos precedentes, el Profesor van den Berg observó en el 2008 que la Convención de Nueva York "necesita ser modernizada" y que muchas de sus disposiciones necesitan ser "añadidas", las disposiciones existentes "no son claras," están "desactualizadas," y necesitan ser "modificadas." 36 En consecuencia, el Profesor van den Berg propuso un "Proyecto Hipotético" de una Convención de Nueva York modificada (el "Proyecto de Convención") diseñado para clarificar, simplificar y modernizar esta convención. ${ }^{37}$

El Proyecto de Convención es de particular interés a la luz de las diferencias entre las Convenciones de Nueva York y de Panamá. Entre sus principales aspectos, el Proyecto de Convención busca clarificar el ámbito de aplicación de la

33. Yer Albert Jan van den Berg, The New York Convention 1958 and Panama Convention 1975: Redundancy or Compatibility?, 5 ARB. INT'L 214, 214-15 (1989) (describiendo cómo la compatibilidad completa "genera la pregunta de cuál podría ser la razón de ser de la Convención de Panamá en vista de la Convención de Nueva York") (traducido al español).

34. Sdem, en p. 229.

35. Ver Albert Jan van den Berg, Striving for Uniform Interpretation, en ENFORCING ARBITRATION AwARDS UNDER THE NEW YORK CONVENTION 41, 42 (United Nations ed., 1999).

36. Ver Albert Jan van den Berg, The Draft Dublin Convention 2008-An Explanation of the Proposed Changes prepared at the Plenary Session of the International Council for Commercial Arbitration ("ICCA") Conference, The New York Convention, p. 50 (10 de junio de 2008), disponible en http://www.arbitration-icea.org/media/0/12133703697430/explanatory_note_ajb_rev06.pdf.

37. Ver Albert Jan van den Berg, Hypothetical Draft Convention on the International Enforcement of Arbitration Agreements and Awards: Text of the Convention (29 de mayo de 2008), disponible en http://www.arbitration-icca.org/media/0/12133674097980/hypothetical_draft_convention_ajbrev06.pdf. 
Convención de Nueva York establecido en el Artículo I. Como se menciona más arriba, la Convención de Panamá carece de tal característica.

El Proyecto de Convención propone derogar el requisito de reciprocidad establecido en el Artículo I(3) de la Convención de Nueva York, y simplifica los requisitos formales para el reconocimiento y ejecución de laudos arbitrales contenidos en el Artículo IV. Éstas son disposiciones que la Convención de Panamá nunca ha contemplado. De acuerdo con el Artículo 2(2)(b) del Proyecto de Convención, las cortes pueden negarse a obligar a las partes a ir a arbitraje si determinan prima facie que no hay un acuerdo arbitral válido de acuerdo con la ley del país donde el laudo será adoptado. Asimismo, el Artículo 3(4) del Proyecto de Convención requiere que las cortes actúen "rápidamente" en una solicitud de ejecución de un laudo arbitral.

A pesar de que mantiene las causales actuales para rechazar el reconocimiento y ejecución contempladas en el Artículo V de la Convención de Nueva York, el Proyecto de Convención sólo aplica estas causales en "casos manifiestos", cuando el asunto haya sido planteado de manera oportuna en el arbitraje subyacente. Por otro lado, el Proyecto de Convención cambia la excepción de política pública que se encuentra en el Artículo V(2)(b) de la Convención de Nueva York (también presente en el Artículo 5(2)(b) de la Convención de Panamá), requiriendo en su lugar una violación del "orden público internacional existente en el país en que se solicita el reconocimiento y la ejecución." 38

Finalmente, el Proyecto de Convención clarifica la discreción de las cortes bajo el Artículo VI de la Convención de Nueva York para suspender acciones de ejecución mientras estén pendientes otros procedimientos de anulacion, y aclara la capacidad de las partes para solicitar la ejecución de laudos arbitrales por medio de otros acuerdos o leyes bajo el Artículo VIl de la

38. tdem. 
Convención de Nueva York. ${ }^{39}$ A pesar que la Convención de Panamá contiene una disposición de suspensión similar en su Artículo 6, no se refiere al reconocimiento y ejecución por medio de otros acuerdos.

El Proyecto de Convención, a pesar de estar dirigido específicamente a defectos identificados en la Convención de Nueva York, también trae importantes implicancias para la Convención de Panamá. El Proyecto de Convención en algunos aspectos disminuiría la brecha entre las dos convenciones a través de la abolición o simplificación de disposiciones de la Convención de Nueva York que no se encuentran en la Convención de Panamá, como por ejemplo la disposición de reciprocidad del Artículo I(3) y los requerimientos formales del Artículo IV.

Al mismo tiempo, ya que también trata temas contenidos en ambas convenciones, el Proyecto de Convención podría ampliar las brechas entre los dos instrumentos, agravando asuntos de compatibilidad y co-existencia. 40

A modo de resumen, hace cincuenta años, fecha del origen de la Convención de Nueva York, América Latina carecía de un marco legal comprensivo para el reconocimiento y ejecución de laudos internacionales. Desde entonces, la mayoría de los estados de América Latina han adoptado las Convenciones de Nueva York y Panamá. A pesar de sus similitudes, las Convenciones de Nueva York y Panamá son instrumentos independientes con diferencias notables. Sin embargo, cada una de las convenciones han contribuido al desarrollo del arbitraje comercial en la región.

39. En particular, el Artículo 7 del Proyecto de Convención dispone que "[s]i un acuerdo arbitral o laudo arbitral puede ser ejecutado sobre una base legal distinta a esta Convención en el país donde el acuerdo o laudo es invocado, la parte que solicita la ejecución puede apoyarse en esa base." (traducido al español). Idem., Art. 7.

40. En relación con la Convención de Panamá, ver, por ejemplo, Claus von Wobeser, The Influence of the New York Convention in Latin America and on the Inter-American Convention on International Commercial Arbitration, 2 DISP. RES. INT'L. 43, 54-55 (2008). 
La jurisprudencia en América Latina sobre arbitraje internacional sigue evolucionando, incluyendo en lo que se refiere a la interpretación y aplicación de las Convenciones de Nueva York y Panamá. Al mismo tiempo, dos tercios de los latinoamericanos todavía desconfían de sus propias cortes..$^{41}$ Esto presagia una continua evolución para el arbitraje comercial en América Latina para las décadas venideras.

41. Informe Latinobarómetro 2007, Corporación Latinobarómetro (2007), http $/ /$ www.latinobarometro.otg. 


\section{Apéndice A.}

\begin{tabular}{|c|c|c|}
\hline \multicolumn{3}{|c|}{$\begin{array}{l}\text { Leyes y Tratados de Arbitraje Comercial } \\
\text { en America Latina } \\
\text { EXTRACTO DEL COMPENOF SOBRE ARBTRRA.JE EN AMERICALATINA }\end{array}$} \\
\hline \multicolumn{3}{|c|}{ 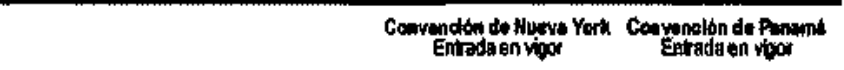 } \\
\hline ARCENTHA & 19091 & 4895 \\
\hline BOLNAA & 1995 & 1999 \\
\hline BADALE & wope & 1095 \\
\hline CHLE & & 1976 \\
\hline COOOHA & 1979 (I) & 697 \\
\hline COSTA RICA & 1988 & t978 \\
\hline CuBA : & 1975 & 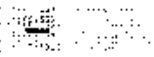 \\
\hline REPUELLICA DOMINICANA & 2002 & - \\
\hline GovapoR & 106 & 1901 \\
\hline EL. SALVADOR & 1998 & 1980 \\
\hline CUNAWWA & 1984 & 1980 \\
\hline HONDURAS & 2004 & 4979 \\
\hline 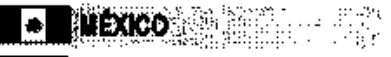 & 1971 & mas \\
\hline MICARAGUA & 2003 & - \\
\hline PAMAW & 1985 & 1976 \\
\hline PARAGUAY & 1998 & 1977 \\
\hline PERO'S & 1968 & 1969 \\
\hline URUGUAY & 1993 & 1977 \\
\hline VEMEZUEA $\mathrm{O}$ ? & 190 & 1985 \\
\hline
\end{tabular}


Apéndice B.

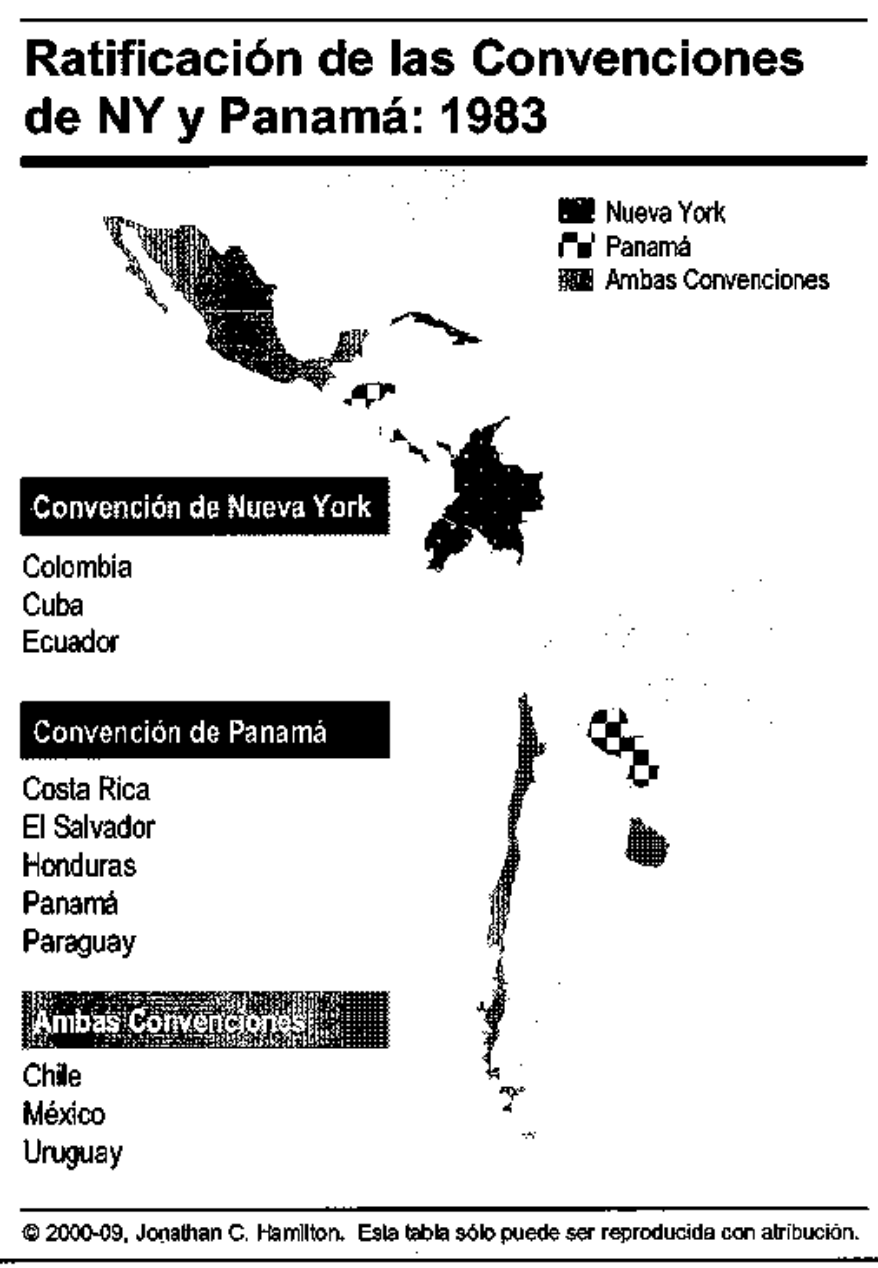


Apéndice C.

\section{Ratificación de las Convenciones de NY y Panamá: 2008}

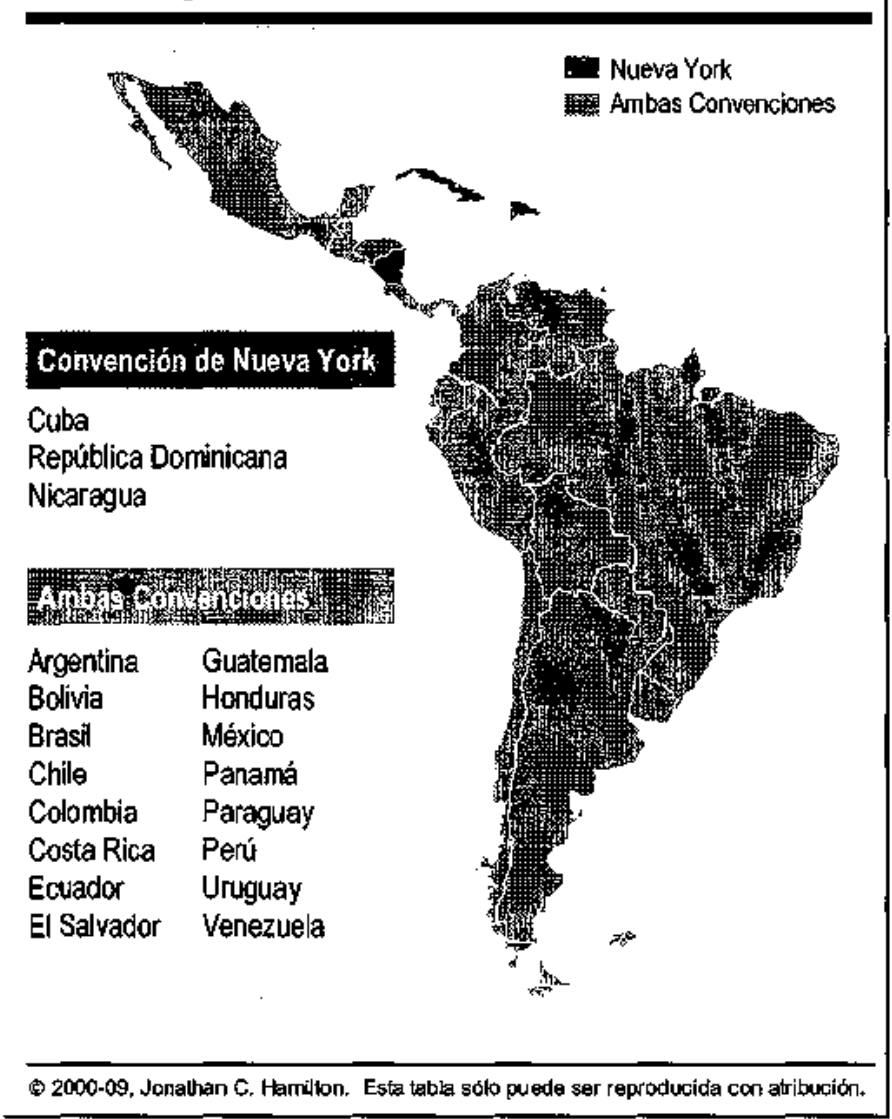


